\title{
Trapping of a random walk by diffusing traps
}

\author{
Frédéric van Wijland \\ Pôle Matière et Systèmes Complexes (CNRS FR2438, Université de Paris VII) and Lab- \\ oratoire de Physique Théorique, CNRS UMR8627, Université de Paris-Sud, 91405 Orsay \\ cedex, France.
}

\begin{abstract}
We present a systematic analytical approach to the trapping of a random walk by a finite density $\rho$ of diffusing traps in arbitrary dimension $d$. We confirm the phenomenologically predicted $\mathrm{e}^{-c_{d} \rho t^{d / 2}}$ time decay of the survival probability, and compute the dimension dependent constant $c_{d}$ to leading order within an $\varepsilon=2-d$ expansion.
\end{abstract}




\section{Introduction}

\subsection{Motivations}

It has been over thirty years that the trapping of a random walk in a medium with absorbing traps is the focus of physicists'attention. The reason is twofold. First and more importantly the experimental relevance of modeling the diffusion of exciton has led to a compact formulation of the problem in terms of trapping of random walks. The second motivation is of theoretical nature. Much is known on the static traps case. Connections between trapping and Lifschitz tails in disordered electronic systems [1], statistics of rare events [2] and Brownian motion theory have been established. That the problem bridges to other areas of physics stems from the ubiquity of random walks and their applications.

Here we will concentrate our study on the diffusion of a random walker in the presence of diffusing traps. The configuration of the traps evolves in time, instead of remaining frozen in its initial distribution. Despite the simplicity of its formulation, this consitutes a genuine many-body problem in which an infinite number of degrees of freedom are coupled: the positions of the traps relative to that of the random walker are correlated.

Let us provide a more precise formulation of the problem we propose to investigate. We are interested in the problem of a tagged walker (hereafter christened as "the walker") evolving in a medium in which a finite density $\rho$ of diffusing traps is present. Both the traps and the tagged walker have the same diffusion

constant. Here we have set the diffusion constant to $\frac{1}{2}$ for aesthetic reasons. However it would be of interest to investigate the diffusion constants ratio dependence, since, as shown in [3], at sufficiently low trap mobility, there exists a rich crossover regime between static and mobile traps. When the walker and a trap meet on the same site then, between $t$ and $t+\mathrm{d} t$, the walker has a probability $\beta \mathrm{d} t$ to die.

The static traps case was "solved" some twenty years ago using field-theoretic methods based on instanton calculus [4, 5, 6] in the sense that the asymptotic behavior of the survival probability has been determined analytically. The onedimensional case can be solved in the sense that the survival probability can be computed exactly by the standard tools of random walk theory [7, 8]. In the meanwhile the mobile traps case has resisted analytic approaches, even in one space dimension, and it is only very recently that extensive numerical studies were devoted to unravelling its fine properties [9]. What can be inferred, however, from phenomenological arguments [11] or from low trap density expansions [12], is that 
the survival probability of the tagged walker decays with time $S$ as

$$
Z(S) \sim \begin{cases}\exp \left(-c_{d} \rho S^{d / 2}\right) & d<2 \\ \exp \left(-c_{d} \rho S\right) & d>2\end{cases}
$$

where $c_{d}$ is a dimension dependent constant (universal in $d<2$, but non universal in $d>2$ ). What makes the problem so analytically difficult to tackle? One answer is that we are dealing with a truly dynamical problem. There is no underlying static partition function, as opposed to the static traps situation. This prevents in particular, the use of quantum field theory methods which proved so successful for static traps. Of course other types of field-theoretic mappings exist (dynamical theories built after the Doi-Peliti mapping), but all of them fail [10] to predict the asymptotic behaviour of the survival probability: just as is the case for static traps, this is a strong coupling problem not accessible with the usual perturbative toolbox. Finally, it is interesting to note the close connection between the present trapping problem and the study of systems of vicious walkers [13] which exhibit some analytic similarities (the one trap problem is the two vicious walker problem).

Our goal in this article is to cast the problem in a form suitable for a systematic analytic treatment. We will go beyond the existing results for the survival probability by setting our calculation in a systematic framework and by giving amplitudes to leading order in an $\varepsilon=2-d$ expansion.

\subsection{Notations}

Consider $N$ random walkers in a volume $V=L^{d}$ and denote by $\rho=\frac{N}{V}$ their average density (eventually the thermodynamic limit $N, V \rightarrow \infty$ with fixed $\rho$ shall be taken). The positions of those walkers are denoted by $\mathbf{x}_{i}(s)$, their initial positions $\mathbf{x}_{i}(0)$ being random in space. Let $\mathbf{r}(s)$ denote the position of the tagged walker, which starts from the origin at time 0 . We employ Brownian motion functionals to describe the dynamics of the system. The action governing the dynamics of the set of $N+1$ walkers is $A_{0}+A_{\text {int }}$, where

$$
A_{0}\left[\mathbf{r},\left\{\mathbf{x}_{i}\right\}\right]=\frac{1}{2} \int_{0}^{S} \mathrm{~d} s\left[\left(\frac{\mathrm{d} \mathbf{r}}{\mathrm{d} s}\right)^{2}+\sum_{i}\left(\frac{\mathrm{d} \mathbf{x}_{i}}{\mathrm{~d} s}\right)^{2}\right]
$$

encodes the free motion of the particles. The interaction term reads

$$
A_{\text {int }}\left[\mathbf{r},\left\{\mathbf{x}_{i}\right\}\right]=\beta \sum_{i} \int_{0}^{S} \mathrm{~d} s \delta^{(d)}\left(\mathbf{r}(s)-\mathbf{x}_{i}(s)\right)
$$


It describes the trapping of $\mathbf{r}$ by the mobile traps $\mathbf{x}_{i}$ 's at a rate $\beta$.

We define two types of averages, that with respect to the free action with an index 0 ,

$$
\langle\ldots\rangle_{0}=\int \mathcal{D} \mathbf{r} \delta^{(d)}(\mathbf{r}(0)) \prod_{i} \int \mathcal{D} \mathbf{x}_{i} \int \frac{\mathrm{d}^{d} x_{i}(0)}{V} \ldots \mathrm{e}^{-A_{0}}
$$

and that with respect to the full interacting process,

$$
\langle\ldots\rangle=\left\langle\ldots \mathrm{e}^{-A_{\text {int }}}\right\rangle_{0}
$$

without any index. Normalization is chosen so that $\langle 1\rangle_{0}=1$, so that $\langle 1\rangle<1$.

\subsection{Quantities of interest}

We define the survival probability after time $S$ by

$$
Z(S) \equiv\langle 1\rangle
$$

and introduce the function $Z(\mathbf{q}, S)$ defined by

$$
Z(\mathbf{q}, S) \equiv\left\langle\mathrm{e}^{i \mathbf{q} \cdot \mathbf{r}(S)}\right\rangle
$$

which allows to define the Fourier transform of the probability of presence a walk conditioned to survive up until time $S$ :

$$
G(\mathbf{q}, S)=\frac{Z(\mathbf{q}, S)}{Z(S)}
$$

From the knowledge of $G(\mathbf{q}, S)$ it should eventually be possible to deduce the mean square displacement $R^{2} \equiv-2 d \frac{\partial G}{\partial q^{2}}$ of a walk conditioned to survive. It is not $a$ priori obvious whether anomalous diffusion should occur and investigating this issue is beyond the scope of the present work.

\subsection{Preliminary analysis}

\subsubsection{Mobile traps, immobile tagged particle}

Here we give the exact result for the survival probability: denote by $f(\mathbf{x}, \tau)$ the probability that a random walk starting from $\mathbf{x}$ reaches the origin for the first time 
exactly at time $\tau$, the survival probability of tagged particle (located at the origin) is given by

$Z(S)=V^{-N} \sum_{\left\{\mathbf{x}_{i}\right\}} \prod_{i=1}^{N}\left(1-\sum_{\tau=0}^{S} f\left(\mathbf{x}_{i}, \tau\right)\right)=\left(1-\frac{1}{V} \sum_{\mathbf{x}} \sum_{\tau} f(\mathbf{x}, \tau)\right)^{N} \stackrel{N \rightarrow \infty}{\simeq} \mathrm{e}^{-\rho \mathcal{N}(S)}$

where $\mathcal{N}(S)\left(\simeq \frac{S^{d / 2}(2 \pi)^{d / 2}}{\Gamma\left(\frac{\varepsilon}{2}\right) \Gamma\left(2-\frac{\varepsilon}{2}\right)}\right.$ if $d<2$ and $\simeq$ if $\left.d>2\right)$ is the average of the number of distinct sites visited by a random walk after $\tau$ steps (a functional derivation of this century old lattice result can be deduced from [14]: $\mathcal{N}(S)=\int \mathrm{d}^{d} x\langle(1-$ $\left.\left.\left.\mathrm{e}^{-\beta \int \mathrm{d} s \delta^{(d)}(\mathbf{r}(s)-\mathbf{x})}\right)\right\rangle_{0}=\beta S \sum_{n \geq 0} \frac{(-1)^{n}\left(\beta S^{\varepsilon / 2}(2 \pi)^{-d / 2} \Gamma(\varepsilon / 2)\right)^{n}}{\Gamma\left(2+n \frac{\varepsilon}{2}\right)}\right)$. This already reproduces the characteristic features of the conjectured behaviour. Note that the result (9) is exact -a property already noticed in [15] - as long as the tagged walker is immobile.

\subsubsection{Dimensionless variables}

It is interesting to note that $d=2$ appears to be the upper critical dimension in this problem. We shall henceforth set $\varepsilon \equiv 2-d$. This can be seen by scaling out the walk's length $S$. We build two independent dimensionless couplings

$$
u \equiv \beta \rho S, \quad v=(4 \pi)^{-d / 2} \rho^{-1} S^{-d / 2}
$$

and further define

$$
w \equiv u v=(4 \pi)^{-d / 2} \beta S^{\frac{\varepsilon}{2}}
$$

The local trap density,

$$
\rho(\mathbf{x}, t) \equiv \sum_{i=1}^{N} \delta^{(d)}\left(\mathbf{x}-\mathbf{x}_{i}(t)\right)
$$

is a Poissonian variable and it is therefore difficult to exactly integrate out its fluctuations. Note that diffusion noise is often well-described by a Gaussian white noise. Such an approximation allows density to become negative (if extremely low probability), and since those very regions of low trap density govern to dynamics, the importance of those unphysical configurations is enhanced which completely messes any analysis based on the Gaussian noise approximation. We are going to attempt an expansion in powers of the trapping probability (which is proportional to $\beta$ ) and eventually take the limit in which this probability goes to 1 . The trapping rate $\beta$ can be viewed as an extra parameter that can be used to probe the model's properties. 


\section{Pertubation expansion for $Z(S)$}

\subsection{General structure of the expansion}

We want to evaluate $Z(\mathbf{q}, S)=\left\langle\mathrm{e}^{i \mathbf{q} \cdot \mathbf{r}(S)}\right\rangle$. First we expand $\mathrm{e}^{-A_{\text {int }}}$ in powers of the trapping rate $\beta$, which yields

$$
\begin{aligned}
\mathrm{e}^{-A_{\text {int }}}= & \sum_{n=0}^{+\infty} \frac{(-\beta)^{n}}{n !} \int_{0}^{S} \mathrm{~d} s_{1} \ldots \mathrm{d} s_{n} \int \frac{\mathrm{d}^{d} k_{1}}{(2 \pi)^{d}} \ldots \frac{\mathrm{d}^{d} k_{n}}{(2 \pi)^{d}} \sum_{i_{1}, \ldots, i_{n}=1}^{N} \\
& \exp \left[i \int_{0}^{S} \mathrm{~d} \tau \mathbf{y}(\tau) \sum_{j=1}^{n} \mathbf{k}_{j} \Theta\left(s_{j}-\tau\right)\right] \\
& \times \exp \left[-i \sum_{j=1}^{n} \int_{0}^{S} \mathrm{~d} \tau \mathbf{y}_{i_{j}}(\tau) \cdot \mathbf{k}_{j} \Theta\left(s_{j}-\tau\right)\right] \\
& \times \exp \left[-i \sum_{j=1}^{n} \mathbf{x}_{i_{j}}(0) \cdot \mathbf{k}_{j}\right]
\end{aligned}
$$

We have denoted by $\mathbf{y}(s)=\frac{\mathrm{d} \mathbf{r}}{\mathrm{d} s}$ the velocity of the tagged walker and by $\mathbf{y}_{i}=$ $\frac{\mathrm{d} \mathbf{x}_{i}}{\mathrm{~d} s}$ that of trap $i$. Consider the $n^{\text {th }}$ order term. It involves an average over $n$ random walkers $\mathbf{x}_{i_{1}}, \ldots, \mathbf{x}_{i_{n}}$. We depict a general $n^{\text {th }}$ order term by the follwoing diagram: In a general diagram, the number of horizontal lines stands for the number of intersections that have take place between times 0 and $S$, this is also the power of $\beta$ in the perturbation expansion. The number of vertical lines, at order $n$, stands for the number of distinct traps that will intersect the tagged walkers's trajectory. A diagram of order $n$ involving $m \leq n$ distinct traps will be proportional to $u^{n} v^{m}=$ $u^{m} w^{n-m}$. It is not a trivial task to determine the proportionality constant; this will depend in a complicated manner on the "topology" of the diagram (the latter constant will be a function of $\varepsilon$ ).

\subsection{An example: diagrams of order 4}

As an example, we explicitly compute the contributions arising from the term $n=$ 4 in the expansion (13). We depict those contributions by a series of diagrams: We now state the explicit evaluation from which one can induct the general properties of those diagrams, such as their leading UV divergence. Diagrams $(a, b, c, d, g)$ exhibit no overlapping loops.

$$
(a)=\frac{u^{4}}{4 !}
$$



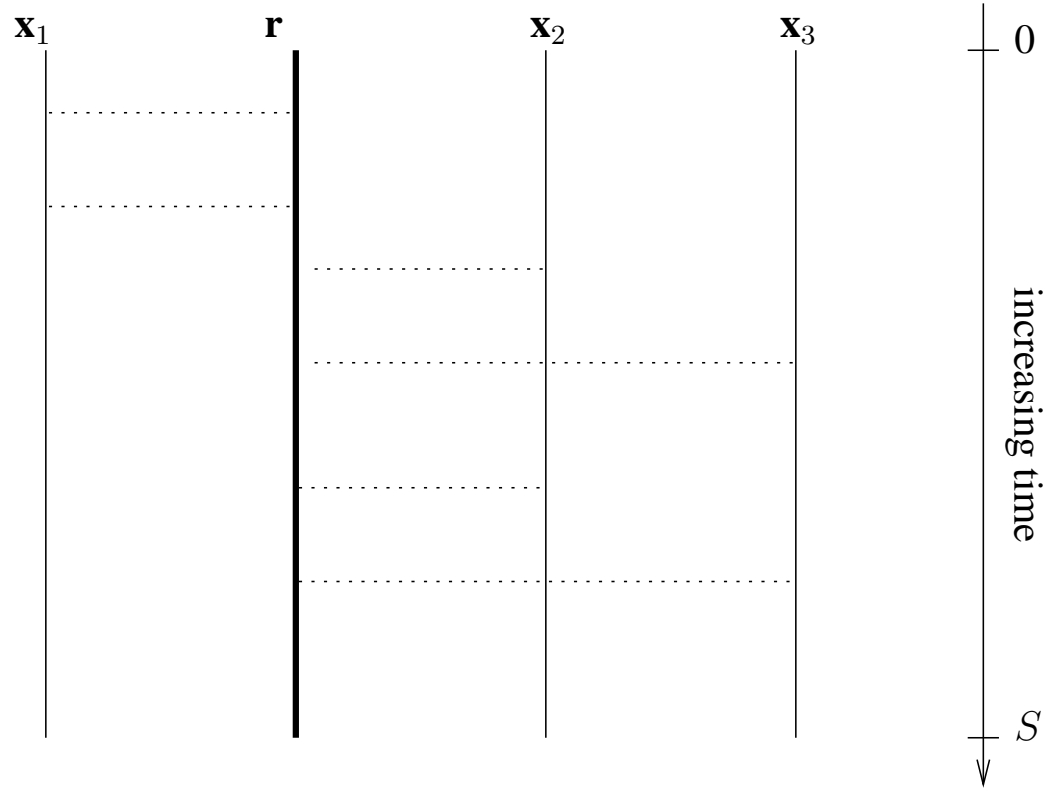

Figure 1: The bold trajectory of the tagged walker $\mathbf{r}$ meets that of the traps $\mathbf{x}_{1}, \mathbf{x}_{2}$, $\mathbf{x}_{3}$. The depicted diagram is of order 6 (there are six intersection times). 

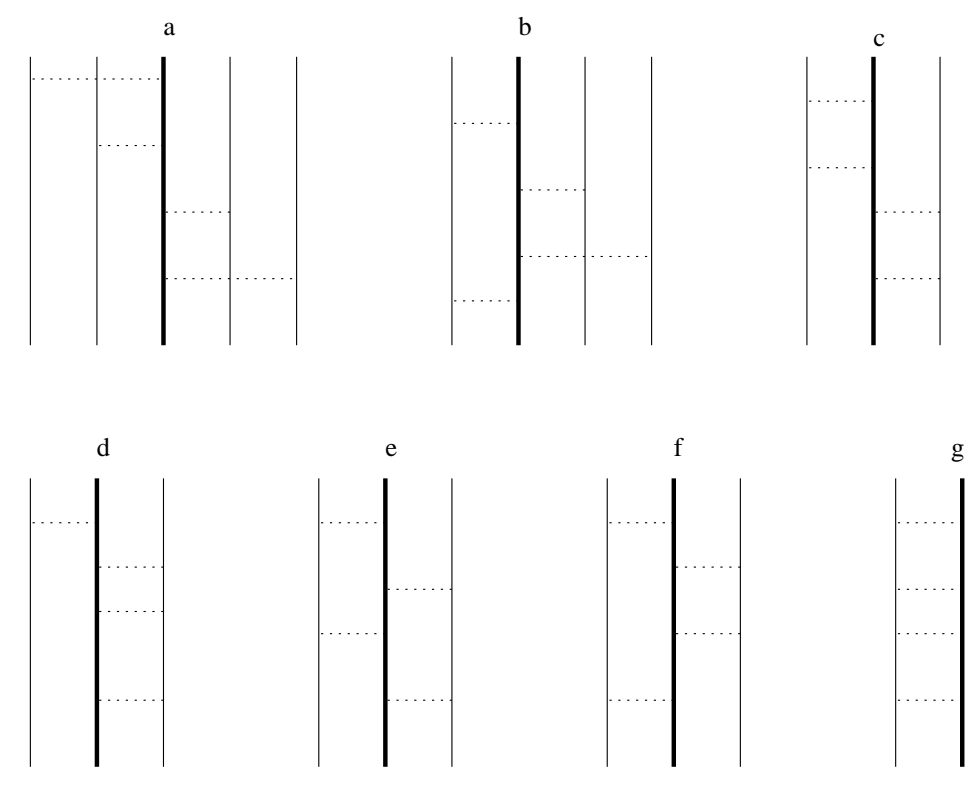

Figure 2: Shown are diagrams of order 4. Diagram (a) is proportional to $u^{4}$, diagram (b) to $u^{3} w$, diagrams (c,d,e,f) to $u^{2} w^{2}$ and diagram (g) to $u w^{3}$.

$$
\begin{aligned}
& (b)=\frac{1}{2} u^{3} w \Gamma(\varepsilon / 2) \frac{1}{\Gamma(2+\varepsilon / 2)} \\
& (c)=\frac{1}{2} u^{2}(w \Gamma(\varepsilon / 2))^{2} \frac{2}{\Gamma(3+\varepsilon)} \\
& (d)=u^{2}(w \Gamma(\varepsilon / 2))^{2} \frac{1}{\Gamma(2+\varepsilon)}
\end{aligned}
$$

Diagrams $(e, f)$ possess an overlapping loop:

$$
\begin{aligned}
(e) & =u^{2} w^{2} \int_{0<x_{1}<x_{3}<x_{2}<x_{4}<1} \mathrm{~d} x_{1} \mathrm{~d} x_{2} \mathrm{~d} x_{3} \mathrm{~d} x_{4}\left(\left(x_{4}-x_{3}\right)\left(x_{2}-x_{1}\right)-\frac{1}{4}\left(x_{2}-x_{3}\right)^{2}\right)^{-\frac{d}{2}} \\
& =\int \mathrm{d} t_{1} \mathrm{~d} t_{2} \mathrm{~d} t_{3} \Theta\left(1-t_{1}-t_{2}-t_{3}\right)\left(1-t_{1}-t_{2}-t_{3}\right)\left(t_{1} t_{2}+t_{2} t_{3}+t_{3} t_{1}+\frac{3}{4} t_{2}^{2}\right)^{-\frac{d}{2}} \\
& =\frac{1}{\varepsilon}\left(-\frac{50}{3}-48 \ln 3+\frac{824}{9} \ln 2-\frac{4}{\sqrt{3}} \ln \frac{2-\sqrt{3}}{2+\sqrt{3}}\right)+\mathcal{O}(1)
\end{aligned}
$$


The coefficient $\frac{3}{4}=\frac{4 D(D+1)}{(2 D+1)^{2}}$, where $D=\frac{1}{2}$ is the trap diffusion constant. If the traps were static, one would have $D=0$ and one would recover a standard $\phi^{4}$ propagator diagram with a double pole in $4-d$. A nonzero diffusion constant acts as a partial UV regulator which shifts the existence of a UV divergence from $d=4$ down to $d=2$.

$$
\begin{aligned}
(f) & =u^{2} w^{2} \int_{0<x_{1}<x_{3}<x_{4}<x_{2}<1} \mathrm{~d} x_{1} \mathrm{~d} x_{2} \mathrm{~d} x_{3} \mathrm{~d} x_{4}\left(x_{4}-x_{3}\right)^{-\frac{d}{2}}\left(x_{2}-x_{1}-\frac{1}{4}\left(x_{4}-x_{3}\right)\right)^{-\frac{d}{2}} \\
& =u^{2} w^{2} \int \mathrm{d} t_{1} \mathrm{~d} t_{2} \mathrm{~d} t_{3} \Theta\left(1-t_{1}-t_{2}-t_{3}\right)\left(1-t_{1}-t_{2}-t_{3}\right) t_{2}^{-\frac{d}{2}}\left(t_{1}+\frac{3}{4} t_{2}+t_{3}\right)^{-\frac{d}{2}} \\
& =u^{2} w^{2}\left(\frac{1}{\varepsilon}+\mathcal{O}(1)\right)
\end{aligned}
$$

Finally,

$$
(g)=u\left(w \Gamma\left(\frac{\varepsilon}{2}\right)\right)^{3} \frac{1}{\Gamma\left(2+3 \frac{\varepsilon}{2}\right)}
$$

The conclusion to be drawn from those explicit expression is the way the shortdistance divergences (the $\varepsilon$ poles) are interwined with the topology of the diagrams which are considered.

\subsection{Nonoverlapping diagrams}

We now focus the subclass of nonoverlapping diagrams: in those diagrams, there are no overlapping loops. In order to fully characterize such a diagram, one needs:

- its order, denoted by $M$; this is also the total number of intersections.

- the number $n$ of distinct traps intersecting the walker's path.

- the number of times $m_{j}$ trap $j(j=1, \ldots, n)$ intersects (in a row) the walker's path. Of course, $\sum_{j=1}^{n} m_{j}=M$.

- among the set $\left\{m_{j}\right\}_{j=1}^{n}$, we count the number of $m_{j}$ 's which are equal. If they take $\nu$ distinct values, we call $p_{\ell}, \ell=1, \ldots, \nu$, the number of $m_{j}$ coefficients that take the same value indexed by $\ell$. This is necessary to properly determine the symmetry coefficient of each diagram. The allowed values of $\nu$ run from 1 through $\nu_{n}=n$ if $n$ can be written in the form $n=k(k+1) / 2$ ( $k$ an integer) and $\nu_{n}=n-1$ otherwise. 


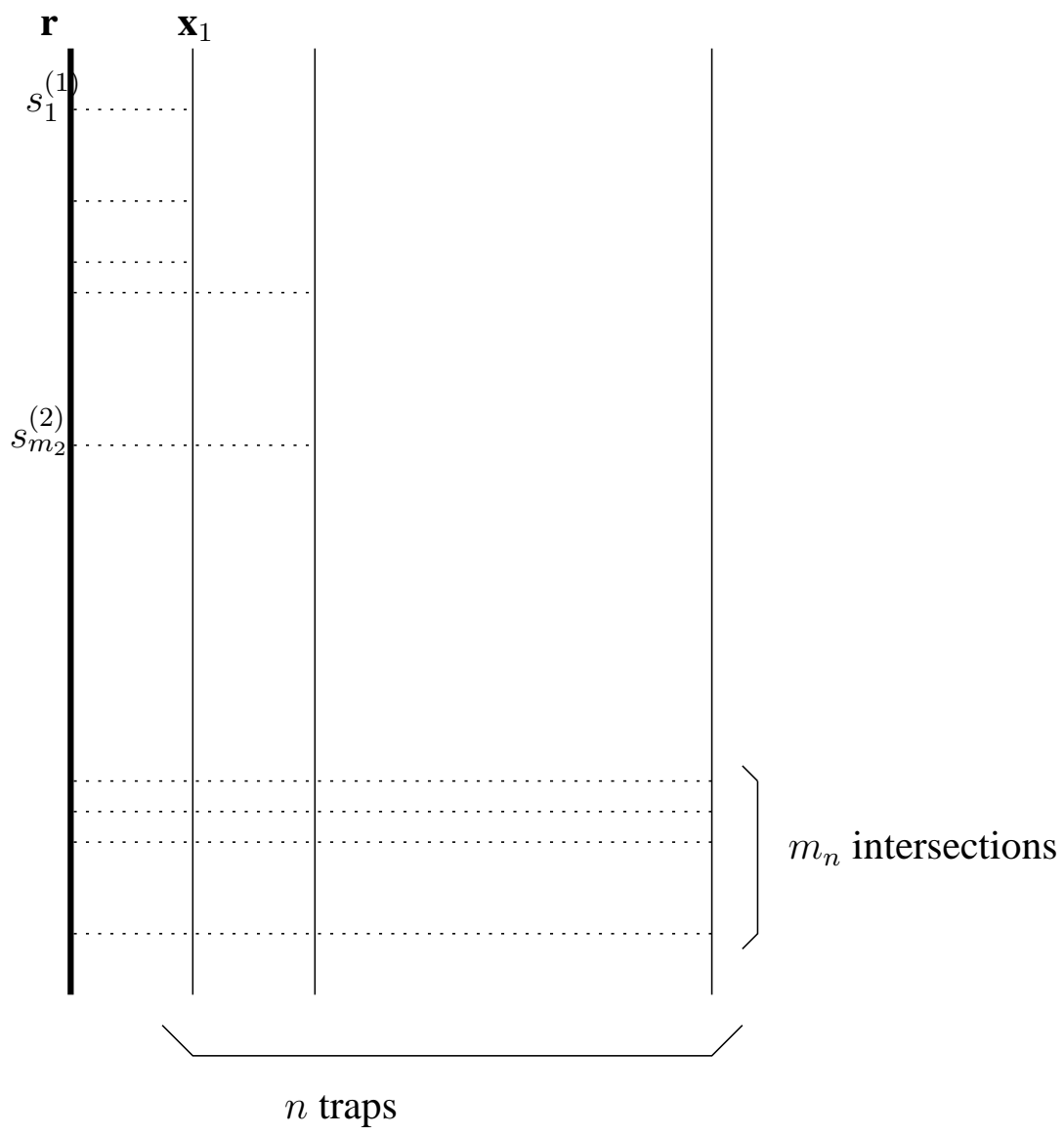

In principle the following calculation holds for $m_{j} \geq 2$ but it can be seen to trivially extend to $m_{j}=1$ without modification.

First consider a particular diagram in which the $M$ intersection times ordered

$$
0 \leq s_{1}^{(1)} \leq s_{2}^{(1)} \leq \ldots \leq s_{m_{1}}^{(1)} \leq s_{1}^{(2)} \leq \ldots \leq s_{m_{n}}^{(n)} \leq S
$$

where the upper index $j(j=1, . ., n)$ denotes the trap that intersects the walker's path and the lower index $i$ counts the number of intersections of trap $j$ ( $i=$ $\left.1 \ldots, m_{j}\right)$. Its graphical representation is as follows: We call $D_{M, n}$ the value of 
that diagram. Given that sequence of intersections, we have to evaluate

$$
\begin{aligned}
D_{M, n}= & \frac{(-\beta)^{M}}{M !} N(N-1) . .(N-n+1) \int \mathrm{d} s_{1}^{(1)} \ldots \mathrm{d} s_{m_{n}}^{(n)} \int \frac{\mathrm{d}^{d} k_{1}^{(1)}}{(2 \pi)^{d}} \ldots \frac{\mathrm{d}^{d} k_{m_{n}}^{(n)}}{(2 \pi)^{d}} \\
& \left\langle\exp \left[i \int_{0}^{S} \mathrm{~d} \tau \mathbf{y}(\tau)\left(\sum_{j=1}^{n} \sum_{i=1}^{m_{j}} \mathbf{k}_{i}^{(j)} \Theta\left(s_{i}^{(j)}-\tau\right)\right)\right]\right. \\
& \exp \left[-i \int_{0}^{S} \mathrm{~d} \tau\left(\sum_{j=1}^{n} \mathbf{y}_{j}(\tau) \sum_{i=1}^{m_{j}} \mathbf{k}_{i}^{(j)} \Theta\left(s_{i}^{(j)}-\tau\right)\right)\right] \\
& \left.\exp \left[-i \sum_{j=1}^{n} \mathbf{x}_{j}(0) \sum_{i=1}^{m_{j}} \mathbf{k}_{i}^{(j)}\right]\right\rangle_{0} \\
& (-1)^{M} \frac{N(N-1) \ldots(N-n+1)}{M^{n}} u^{n} w^{M-n} \\
& \times \int_{0}^{1} \mathrm{~d} t_{1} \int_{0}^{1-t_{1}} \mathrm{~d} t_{2} \ldots \int_{0}^{1-t_{1} \ldots-t_{M-1}} \mathrm{~d} t_{M} \\
& \left(t_{1}^{0} t_{2}^{-\frac{d}{2}} t_{3}^{-\frac{d}{2}} \ldots t_{m_{1}+1}^{0} t_{m_{1}+2}^{-\frac{d}{2}} \ldots t_{m_{1}+\ldots+m_{n-1}+1}^{0} \ldots t_{M}^{-\frac{d}{2}}\right) \\
= & \frac{(-1)^{M}}{M !} \frac{N(N-1) . .(N-n+1)}{N^{n}} \frac{u^{n}\left(w \Gamma\left(\frac{\varepsilon}{2}\right)\right)^{M-n}}{\Gamma\left(n+1+(M-n) \frac{\varepsilon}{2}\right)}
\end{aligned}
$$

While the explicit evaluation is not a trivial task, the final result is particularly simple.

We now consider a general diagram of order $M$ with $n$ intersecting traps characterized by $\left\{m_{j}\right\}_{j=1, \ldots, n},\left\{p_{\ell}\right\}_{\ell=1, \ldots, \nu}$. We do not specify the order in which the traps intersect the walker's path. Such a diagram has a value

$\frac{M !}{\prod_{j=1}^{n} m_{j} ! \prod_{\ell=1}^{\nu} p_{\ell} !} n !\left(\prod_{j=1}^{n} m_{j} !\right) D_{M, n}=(-1)^{M} \frac{n !}{\prod_{\ell=1}^{\nu} p_{\ell} !} \frac{u^{n}\left(w \Gamma\left(\frac{\varepsilon}{2}\right)\right)^{M-n}}{\Gamma\left(n+1+(M-n) \frac{\varepsilon}{2}\right)}$

At a given order $M$, a diagram involving $n$ distinct walkers has a higher $\varepsilon$-divergence if it is free of overlapping loops. Retaining for each order $M$ only the nonoverlap- 
ping diagrams, we get the survival probabiilty

$$
Z(S)=1+\sum_{M=1}^{+\infty} \sum_{n=1}^{M} \sum_{m_{1}+\ldots+m_{n}=M} \sum_{\nu=1}^{M} \sum_{p_{1}+\ldots+p_{\nu}=n}(-1)^{M} \frac{n !}{\prod_{\ell=1}^{\nu} p_{\ell} !} \frac{u^{n}\left(w \Gamma\left(\frac{\varepsilon}{2}\right)\right)^{M-n}}{\Gamma\left(n+1+(M-n) \frac{\varepsilon}{2}\right)}
$$

Since we are concerned with leading divergences, we will approximate

$$
\frac{1}{\Gamma\left(n+1+(M-n) \frac{\varepsilon}{2}\right)} \simeq \frac{1}{n !}
$$

Summing over all values of $M-n$ yields a factor $\left(1+\frac{w}{\Gamma\left(\frac{\varepsilon}{2}\right)}\right)^{-n}$ Setting $g \equiv$ $\frac{u}{1+w \Gamma\left(\frac{\varepsilon}{2}\right)}$, we are left with

$$
Z(S)=\mathrm{e}^{-g}
$$

In the limit $u, w \rightarrow \infty$ with $\frac{u}{w}$ fixed, this leads to the asymptotic behaviour

$$
Z(S) \sim \mathrm{e}^{-c_{\varepsilon} \rho S^{d / 2}}
$$

with $c_{\varepsilon}=2 \pi \varepsilon+\mathcal{O}\left(\varepsilon^{2}\right)$. We emphasize that this is not a low density expansion since the variable $\frac{u}{w} \propto \rho S^{d / 2}$ is held constant. We could also have written

$$
Z(S) \sim \exp \left[-u \sum_{n=0}^{\infty} \frac{\left(-w \Gamma\left(\frac{\varepsilon}{2}\right)\right)^{n}}{\Gamma\left(2+n \frac{\varepsilon}{2}\right)}\right]
$$

which is, to leading order in $\varepsilon$, equivalent to the previously mentioned result.

The only approximation made is to neglect overlapping diagrams. This is equivalent to performing an $\varepsilon$-expansion of $c_{\varepsilon}$. Compare two diagrams that differ by the presence of an overlapping loop instead of a non-overlapping loop: in the latter, the leading short distance singularity is not constrained by time ordering. In the former, the time ordering associated with an overlapping loop acts as a partial short-distance regulator. The related UV divergence will always be smoother than in the nonoverlapping counterpart. However the power of $S$ that appears in Eq. (27) is exact since, at a given order in $u$ and $w$, diagrams with or without overlapping loops have the same $S$ dependence. We argue that a diagram containing at least a pair of overlapping loops has a softer $\varepsilon$ divergence than the equivalent diagram in which the overlapping loops have been disentangled. 


\subsection{Perturbation expansion of $Z(\mathbf{q}, S)$}

As before, we shall now keep in the perturbation expansion only diagrams containing no loop overlap, this leads to

$$
\begin{aligned}
D_{M, n}= & \frac{(-\beta)^{M}}{M !} N(N-1) . .(N-n+1) \int \mathrm{d} s_{1}^{(1)} \ldots \mathrm{d} s_{m_{n}}^{(n)} \int \frac{\mathrm{d}^{d} k_{1}^{(1)}}{(2 \pi)^{d}} \ldots \frac{\mathrm{d}^{d} k_{m_{n}}^{(n)}}{(2 \pi)^{d}} \\
& \left\langle\exp \left[i \int_{0}^{S} \mathrm{~d} \tau \mathbf{y}(\tau)\left(\mathbf{q} \Theta(S-\tau)+\sum_{j=1}^{n} \sum_{i=1}^{m_{j}} \mathbf{k}_{i}^{(j)} \Theta\left(s_{i}^{(j)}-\tau\right)\right)\right]\right. \\
& \exp \left[-i \int_{0}^{S} \mathrm{~d} \tau\left(\sum_{j=1}^{n} \mathbf{y}_{j}(\tau) \sum_{i=1}^{m_{j}} \mathbf{k}_{i}^{(j)} \Theta\left(s_{i}^{(j)}-\tau\right)\right)\right] \\
= & \frac{(-1)^{M}}{M !} \mathrm{e}^{-\frac{q^{2} S}{2}} \frac{N(N-1) \ldots(N-n+1)}{M^{n}} u^{n} w^{M-n} \\
& \times \int_{0}^{1} \mathrm{~d} t_{1} \int_{0}^{1-t_{1}} \mathrm{~d} t_{2} \ldots \int_{0}^{1-t_{1} \ldots-t_{M-1}} \mathrm{~d}_{M} \\
& \left(t_{1}^{0} t_{2}^{-\frac{d}{2}} \mathrm{e}^{\frac{q^{2} S t_{2}}{4}} t_{3}^{\frac{d}{2}} \mathrm{e}^{\frac{q^{2} S t_{3}}{4}} \ldots t_{m_{1}+1}^{0} t_{m_{1}+2}^{-\frac{d}{2}} \mathrm{e}^{\frac{q^{2} S t_{m_{1}+2}}{4}} \ldots t_{m_{1}+\ldots+m_{n-1}+1}^{0} \ldots t_{M}^{-\frac{d}{2}} \mathrm{e}^{\frac{q^{2} S t_{M}}{4}}\right) \\
= & \mathrm{e}^{-\frac{q^{2} S}{4}} \frac{(-1)^{M}}{M !} u^{n}\left(w \Gamma\left(\frac{\varepsilon}{2}\right)\right)^{M-n} \int \frac{\mathrm{d} z}{2 \pi i} \mathrm{e}^{z} \frac{1}{\left(z+\frac{q^{2} S}{4}\right)^{n+1}} z^{-(M-n) \frac{\varepsilon}{2}}
\end{aligned}
$$

where the integration path runs from $-i \infty+0$ up to $+i \infty+0$. The final result now reads

$$
Z(\mathbf{q}, S)=\mathrm{e}^{-\frac{q^{2} S}{4}} \int \frac{\mathrm{d} z}{2 \pi i} \mathrm{e}^{z} \int_{0}^{+\infty} \mathrm{d} x \mathrm{e}^{-x\left(z+\frac{q^{2} S}{4}\right)} \exp \left[-\frac{u x}{1+\frac{w}{\Gamma(\varepsilon / 2) z^{\varepsilon / 2}}}\right]
$$

so that $G(\mathbf{q}, S)=\mathrm{e}^{-\frac{q^{2} S}{2}}$. Diffusion is not affected at this level of the approximation.

\section{Discussion}




\subsection{Comparison with the static trap problem}

When traps are static, the Brownian motion functional depends on the tagged walkers trajectory only through its local time $\chi(\mathbf{x}, S) \equiv \int_{0}^{S} \mathrm{~d} s \delta^{(d)}(\mathbf{r}(s)-\mathbf{x})$, hence it can be mapped onto an $O(n)$ field theory with an interaction term than can be shown to be

$A_{\text {eff }}[\mathbf{r}]=-\int \mathrm{d}^{d} x \ln \left(1-\rho+\rho \mathrm{e}^{-\beta \int_{0}^{S} \mathrm{~d} s \delta^{(d)}(\mathbf{r}(s)-\mathbf{x})}\right) \rightarrow-\int \mathrm{d}^{d} x \ln \left(1-\rho+\rho \mathrm{e}^{-\beta \phi^{2}}\right)$

We refer the reader to [ $[$, 5, 6] and references therein for details on how to derive this correspondence and how to exploit it. For small values of $\beta$ or of the field this produces an attractive $-\phi^{4}$ theory (which has no minimum, and which is equivalent to truncating the walker's effective self-interation potential to that of a selfattracting walk $-\beta^{2} \rho^{2} \int \mathrm{d} s \mathrm{~d} s^{\prime} \delta^{(d)}\left(\mathbf{r}(s)-\mathbf{r}\left(s^{\prime}\right)\right)$ ), hence the complete knowledge of the interaction potential is necessary. No truncation of the interaction potential can be used.

Attempting an expansion in powers of $\beta$ will generate self-interaction diagrams that must be evaluated in a standard way (a loop corresponds to a $\delta\left(\mathbf{r}(s)-\mathbf{r}\left(s^{\prime}\right)\right)$ interaction). Loops in diagrams occur when a trap is visited at least twice. All those features have their counterpart when traps are mobile.

If traps are mobile, the best Langevin equation that describes the density of the diffusing traps is

$$
\partial_{t} \rho-D \Delta \rho=\eta, \quad\left\langle\eta(\mathbf{x}, t) \eta\left(\mathbf{x}^{\prime}, t^{\prime}\right)\right\rangle=2 D \rho \nabla \nabla^{\prime} \delta\left(t-t^{\prime}\right) \delta^{(d)}\left(\mathbf{x}-\mathbf{x}^{\prime}\right)
$$

If this Langevin equation were exact (which it is not since the density is a Poissonian variable and not a Gaussian one), this would lead to an effective interaction term depending only upon the path $\mathbf{r}$

$$
A_{\mathrm{eff}}[\mathbf{r}]=-\beta^{2} \rho^{2} \int_{0}^{S} \mathrm{~d} s \mathrm{~d} s^{\prime} \frac{1}{\left(4 \pi D\left|s-s^{\prime}\right|\right)^{d / 2}} \exp \left[-\frac{\left(\mathbf{r}(s)-\mathbf{r}\left(s^{\prime}\right)\right)^{2}}{4 D\left|s-s^{\prime}\right|}\right]
$$

We now see that the $\delta^{(d)}\left(\mathbf{r}(s)-\mathbf{r}\left(s^{\prime}\right)\right)$-function appearing in the static traps case has been replaced by $g\left(\mathbf{r}(s)-\mathbf{r}\left(s^{\prime}\right)\right)$ (with $g(\mathbf{x}, \tau) \equiv(4 \pi D|\tau|)^{-d / 2} \mathrm{e}^{-\mathbf{x}^{2} /(4 D|\tau|)}$ and $D=1 / 2)$. In fact, any given graph of the static traps problem has its mobile traps counterpart, which is evaluated simply by replacing the $\delta$-function in the loops (whether overlapping or not) by the traps propagator $g$. There is a one-to-one correspondence between the graphs of the two problems. 


\subsection{Beyond the leading order}

For static traps the mean-square displacement is known [阿] to scale as $R^{2} \sim S^{\frac{d+1}{d+2}}$ and the return to the origin probability as $S^{-\frac{d}{d+2}}$. Within the framework of our approximation, if traps are diffusing, we find that both the mean-square displacement and the return to the origin probability take their simple random walk asymptotics. Expanding the function $G(\mathbf{q}, S)$ beyond leading order in $\varepsilon$ shows that this picture might not hold for the full interacting theory. Indeed, the neglected UV divergencies could be responsible for a modified scaling behavior. For instance, one can show that $G(\mathbf{q}, S)=\mathrm{e}^{-\frac{q^{2} S}{2}}\left(1+\frac{u^{4} v^{2}}{\varepsilon} F\left(q^{2} S\right)+\mathcal{O}(1)\right)$, with $F$ a known function that can easily be expanded in the vicinity of 0 . Deeper insight into the structure of the perturbation expansion is required before exploiting such results in the spirit of the approach developped by Duplantier for polymers [16].

\subsection{Conclusion}

We have given the first systematic approximation scheme for the survival probability of a random walk evolving in a medium infested with freely diffusing traps. Our method exploits the properties of low-dimensional Brownian motion. As opposed to the static trap case, no mapping to an equilibrium-like field theory exists and due to the strong coupling nature of the problem the elegant field-theoretic methods of reaction-diffusion problems fail. Extending the Brownian motion formalism to truly nonequilibrium processes therefore proved unexpectedly fruitful. The method which we have elaborated should now be put to the test on a series of related problems which we now list. The first question that comes to mind is concerned with the scaling properties of a surviving path: what is the scaling behaviour of the walker's mean-square displacement between 0 and $S$, provided it has survived up until that time. Questions of lesser importance concern the number of distinct sites visited by the walker, its return to the origin probability or the algebraic area swept (still restraining the average to surviving paths). Numerical approaches are usually difficult to implement [9] when it comes to exploring those properties due to the poor statistics over surviving events. Future work should address those issues.

Acknowledgments : The author would like to thank Henk Hilhorst and Bertrand Duplantier for repeated discussions on this work. 


\section{References}

[1] J.M. Luttinger and R.C. Tao, Ann. Phys. (NY) 145 (1983) 185.

[2] M.D. Donsker and S.R.S. Varadhan, Commun. Pure Appl. Math. 28 (1975) 525 .

[3] A. M. Berezhkovskii, Yu. A. Makhnovskii and R. A. Suris, J. Phys. A 22 (1989) L615.

[4] T. C. Lubensky, Phys. Rev. A 30 (1984) 2657.

[5] S. Renn, Nucl. Phys. B 275 [FS17] (1986) 273.

[6] Th. M. Nieuwenhuizen, Phys. Rev. Lett. 62 (1989) 357.

[7] G. H. Weiss and S. Havlin, J. Stat. Phys. 37 (1984) 17.

[8] J.-M. Luck, Systèmes désordonnés unidimensionnels (Aléa Saclay 1992).

[9] V. Mehra and P. Grassberger, preprint cond-mat 0111124.

[10] K. Oerding and F. van Wijland, unpublished.

[11] S. Redner and K. Kang, J. Phys. A 17 (1984) L451.

[12] A. Szabo, R. Zwanzig and N. Agmon, Phys. Rev. Lett. 61 (1988) 2496.

[13] S.Mukherji and S.M. Bhattacharjee, J. Phys. A 26 (1993) L1139.

[14] B. Duplantier, Phys. Rev. Lett. 62 (1989) 2337.

[15] A. Blumen, G. Zumofen and J. Klafter, Phys. Rev. B 30 (1984) 5379.

[16] B. Duplantier, J. Physique 47 (1986) 569. 